\title{
IMMUNOHISTOCHEMICAL STUDY OF CYTOCHROME C OXIDASE WITH A MONOCLONAL ANTIBODY IN MITOCHONDRIAL MYOPATHY
}

\author{
Itsuro HIGUCHI, Masanori NAKAGAWA, Mitsuhiro OSAME, \\ TAKashi YAMANO aNd Hidetoshi FUKUNAGA
}

The Third Department of Internal Medicine, Faculty of Medicine, Kagoshima University, 1208-1, Usuki-cho, Kagoshima 890

Received for publication November 13, 1987 and in revised form February 9, 1988

\begin{abstract}
Immunohistochemical examinations using monoclonal antibody against subunit IV of cytochrome c oxidase were carried out in the muscle of patients with various mitochondrial myopathies having histochemical partial deficiency of cytochrome c oxidase activity. The ragged red fibers showed positive reaction for the monoclonal antibody even in the histochemical cytochrome $c$ oxidase deficient fibers. However, in the histochemical cytochrome $c$ oxidase deficient fibers with normal histological appearance, the immunohistochemical reaction varied. While all fibers with immunohistochemical deficient reactivity for subunit IV of cytochrome $c$ oxidase had no detectable histochemical cytochrome c oxidase activity, subunit IV protein deficiency was not the cause of all the histochemical cytochrome c oxidase deficient fibers.
\end{abstract}

Total deficiency of cytochrome c oxidase (CCO) has been well studied $(3,7,15)$ and reviewed (4) in biopsied muscle specimens.

Recently, suggestive evidence of partial CCO deficiency in the skeletal muscle of some cases of mitochondrial myopathy have been shown histochemically (9-11). While several immunohistochemical studies with polyclonal antibody of CCO have been previously reported $(1,2,12,16)$, we are not aware of any immunohistochemical study using monoclonal antibody against the subunit protein of CCO. Hence this study was intended for immunohistochemical examination using monoclonal antibody against subunit IV of CCO on cases with mitochondrial myopathy having histochemical partial deficiency of CCO.

\section{MATERIALS AND METHODS}

Included were 6 patients having various forms of mitochondrial myopathy. Their clinical summaries are presented in Table 1.

The biopsied muscle specimens were frozen in isopentane cooled with liquid nitrogen. Serial 10 micron frozen sections were stained with hematoxylin and eosin, modified Gomori trichrome, reduced nicotinamide adenine dinucleotide tetrazolium reductase (NADH-TR) and adenosine triphosphatase by the methods described by Dubowitz and Brooke (5). To demonstrate the histochemical CCO activity, the 
Higuchi et al.

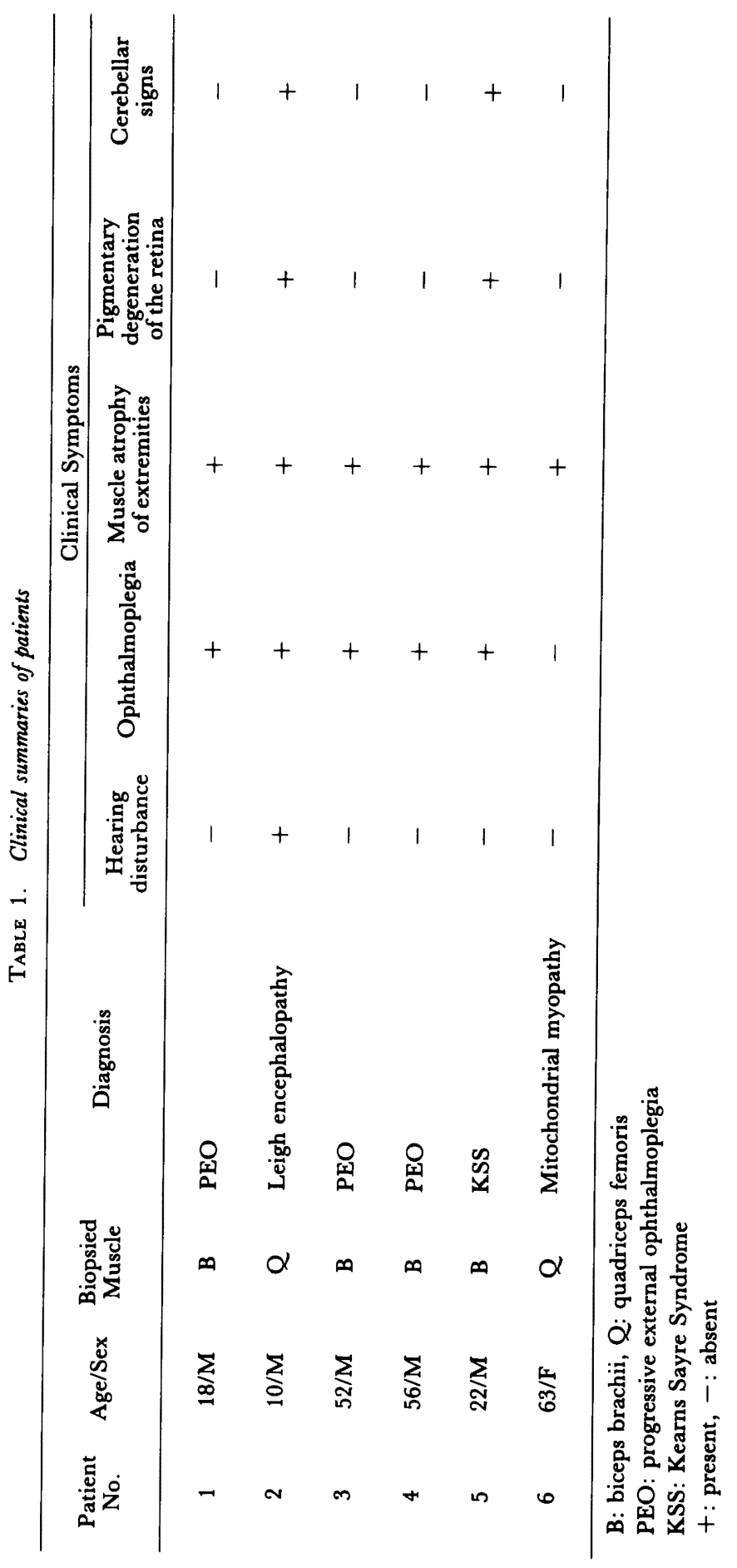




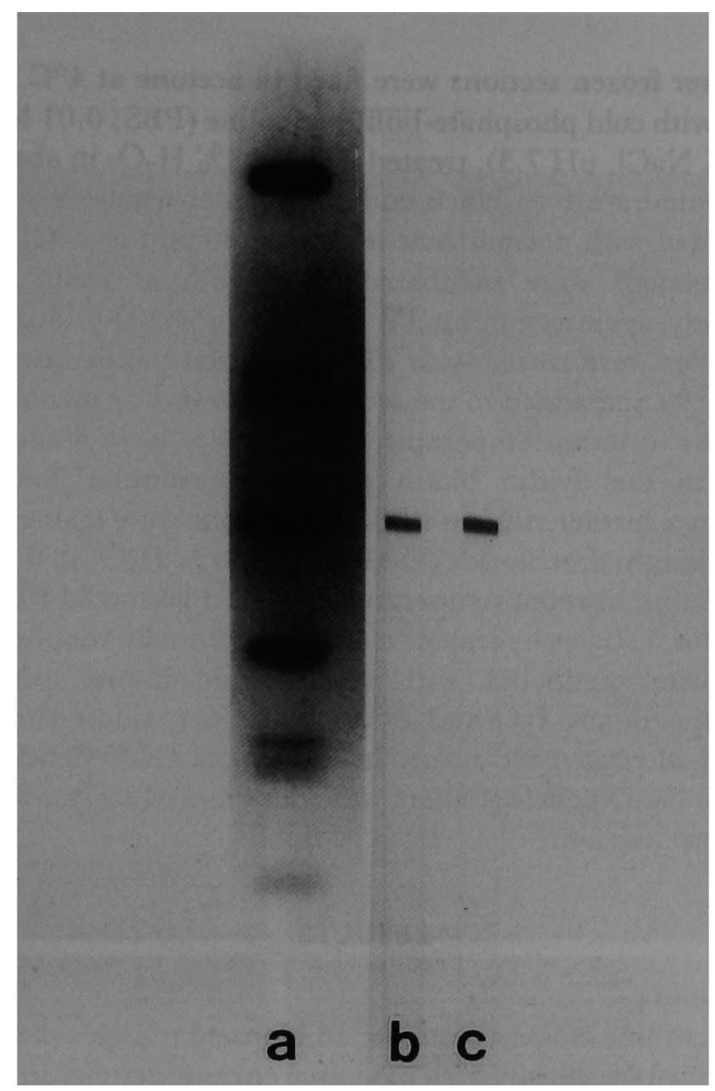

FIG. 1. Specificity of the monoclonal antibody. a. SDS-PAGE of human heart CCO; the gel was stained with Coomassie Blue. CCO subunits are indicated. b. Human heart CCO was separated by SDS-PAGE and transferred to nitrocellulose membrane. The membrane was reacted with the monoclonal antibody diluted to 1:10000. c. Human skeletal muscle CCO was separated and stained in the same manner as $b$.

frozen sections were incubated in the staining solution for one $\mathrm{hr}$ at $37^{\circ} \mathrm{C}$ using the method of Seligman et al. (14).

CCO purification and preparation of hybridomas were made employing the method described by one of the authors (13). Skeletal muscle mitochondria was isolated from autopsied iliopsoas muscle and CCO was purified using the same method as that for heart CCO. The hybridomas were screened by enzyme linked immunosorbent assay (ELISA) and western blot as previously described (13). The antibody that reacted with both heart and skeletal muscle CCO subunit IV was selected (Fig. 1).

Immunohistochemical investigation of CCO was carried out on frozen sections using the $\mathrm{ABC}$ immunoperoxidase method (8). Avidin, biotinylated peroxidase and affinity purified biotinylated goat anti-mouse IgG (Vectastain ABC kit) were obtained from Vector Laboratories (Burlingame, CA). The details of the procedure were as 
follows.

Ten micrometer frozen sections were fixed in acetone at $4^{\circ} \mathrm{C}$ for $30 \mathrm{~min}$. They were then washed with cold phosphate-buffered saline (PBS; $0.01 \mathrm{M}$ phosphate buffer containing $0.085 \% \mathrm{NaCl}, \mathrm{pH} 7.3$ ), treated with $0.03 \% \mathrm{H}_{2} \mathrm{O}_{2}$ in absolute methanol for $30 \mathrm{~min}$ at room temperature to block endogenous peroxidase activity, washed with cold PBS, and treated with normal goat serum for $30 \mathrm{~min}$ at $4^{\circ} \mathrm{C}$. After removal of the serum, the sections were incubated for $30 \mathrm{~min}$ at room temperature with monoclonal antibody against subunit IV of CCO at 1:1000 dilution in a humidity chamber. The slides were rinsed with PBS, and then biotinylated goat anti-mouse IgG was diluted 1:200 and added to the sections, followed by incubation for $30 \mathrm{~min}$ in a humidity chamber at room temperature. The slides were washed with cold PBS, and then rinsed in the avidin biotin peroxidase complex for $30 \mathrm{~min}$ at room temperature. After a further rinse in PBS, the sections were stained with $0.05 \%$ 3, $3^{\prime}$ diaminobenzidine tetrahydrochloride (DAB) plus $0.01 \% \mathrm{H}_{2} \mathrm{O}_{2}$ in $0.05 \mathrm{M}$ tris- $\mathrm{HCl}$ buffer ( $\mathrm{pH}$ 7.6) for $10 \mathrm{~min}$ at room temperature, washed with cold PBS, counterstained with hematoxylin for $5 \mathrm{sec}$, dehydrated, cleared, and finally mounted. As a control, frozen sections were incubated with nonimmune mouse serum followed by biotinylated goat anti-mouse IgG and avidin biotin peroxidase complex.

The frequency of ragged red fibers, histochemical CCO deficient fibers and immunohistochemical CCO deficient fibers were determined by randomly counting 400 muscle fibers in each section.

\section{RESULTS}

Histochemical Staining

In Gomori trichrome-stained sections, all biopsied muscles of 6 patients contained ragged red fibers which showed high oxidative enzyme activity in NADH-TR staining. The frequency of ragged red fibers varied from 0.5 to $17 \%$ (Table 2). In CCO reacted sections, all biopsied muscles showed histochemical partial deficiency of CCO activity. As compared with ATPase reacted sections, the CCO deficient fibers consisted of both histochemical fiber types. The frequency of histochemical CCO defcient fibers varied from 2 to $48 \%$ (Table 2). Histochemical CCO deficient fibers were seen not only in degenerating fibers but also in histologically normal fibers (Figs. 2, 3). The latter consisted of both histochemical fiber types. The histochemical CCO

TABLE 2. Frequency of ragged red fibers, histochemical CCO deficient fibers and immunohistochemical CCO deficient fibers

\begin{tabular}{cccc}
\hline \multirow{2}{*}{$\begin{array}{c}\text { Patient } \\
\text { No. }\end{array}$} & $\begin{array}{c}\text { Ragged Red } \\
\text { Fibers (\%) }\end{array}$ & Histochemistry & Immunohistochemistry \\
\cline { 3 - 4 } 1 & 5 & 16 & 3 \\
2 & 13 & 48 & 9 \\
3 & 1 & 25 & 4 \\
4 & 17 & 5 & 0 \\
5 & 0.5 & 2 & 1 \\
6 & 4 & 3 & 0 \\
\hline
\end{tabular}



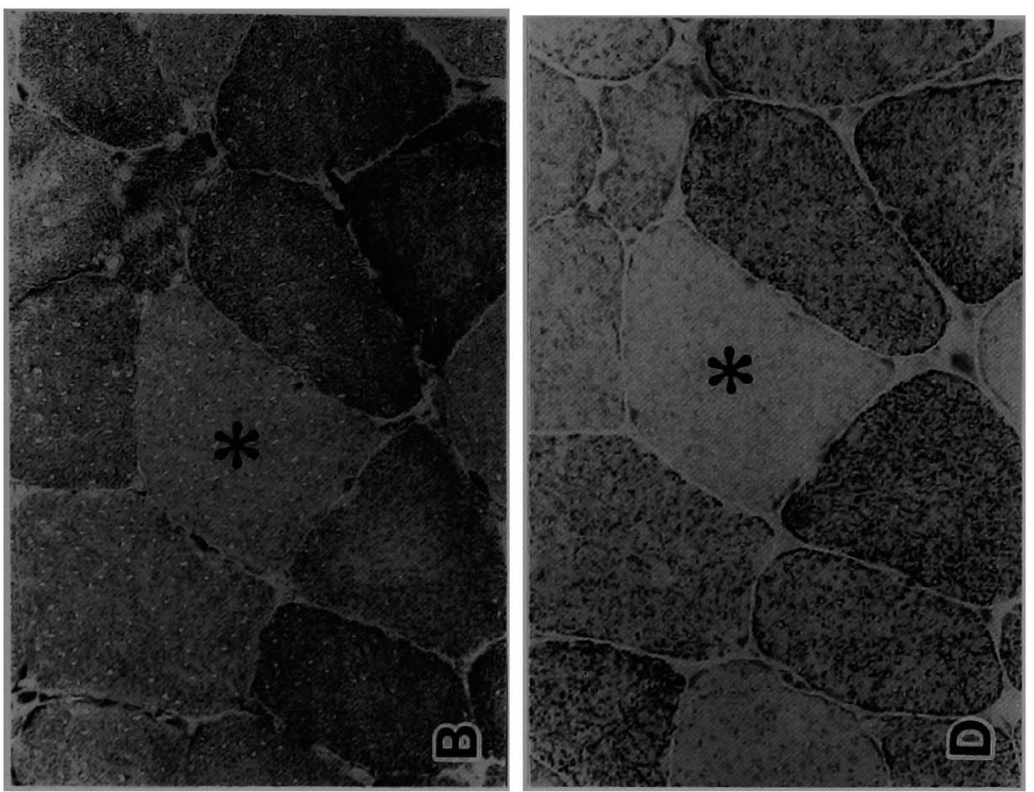

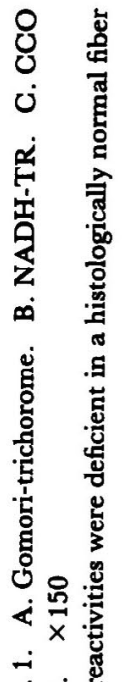
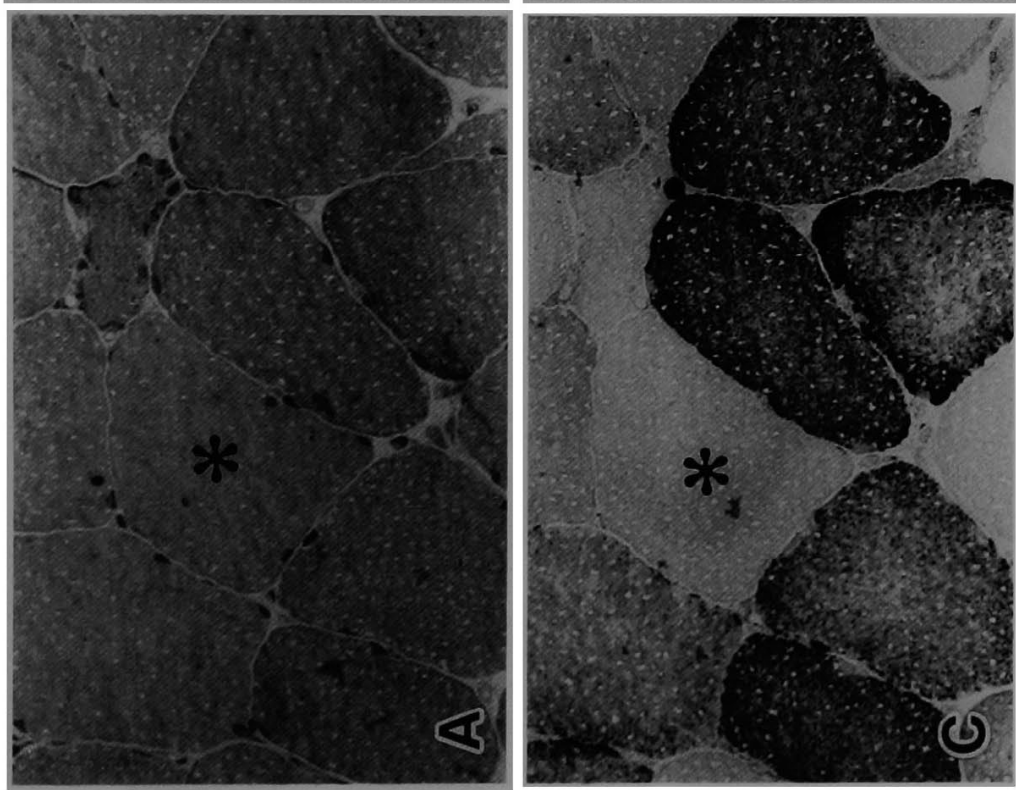

之ें

要

羟

宊

녕

要

글

过声

ब용

○.

穴

ส

顿宣

존

造

究鱼

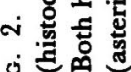

它 


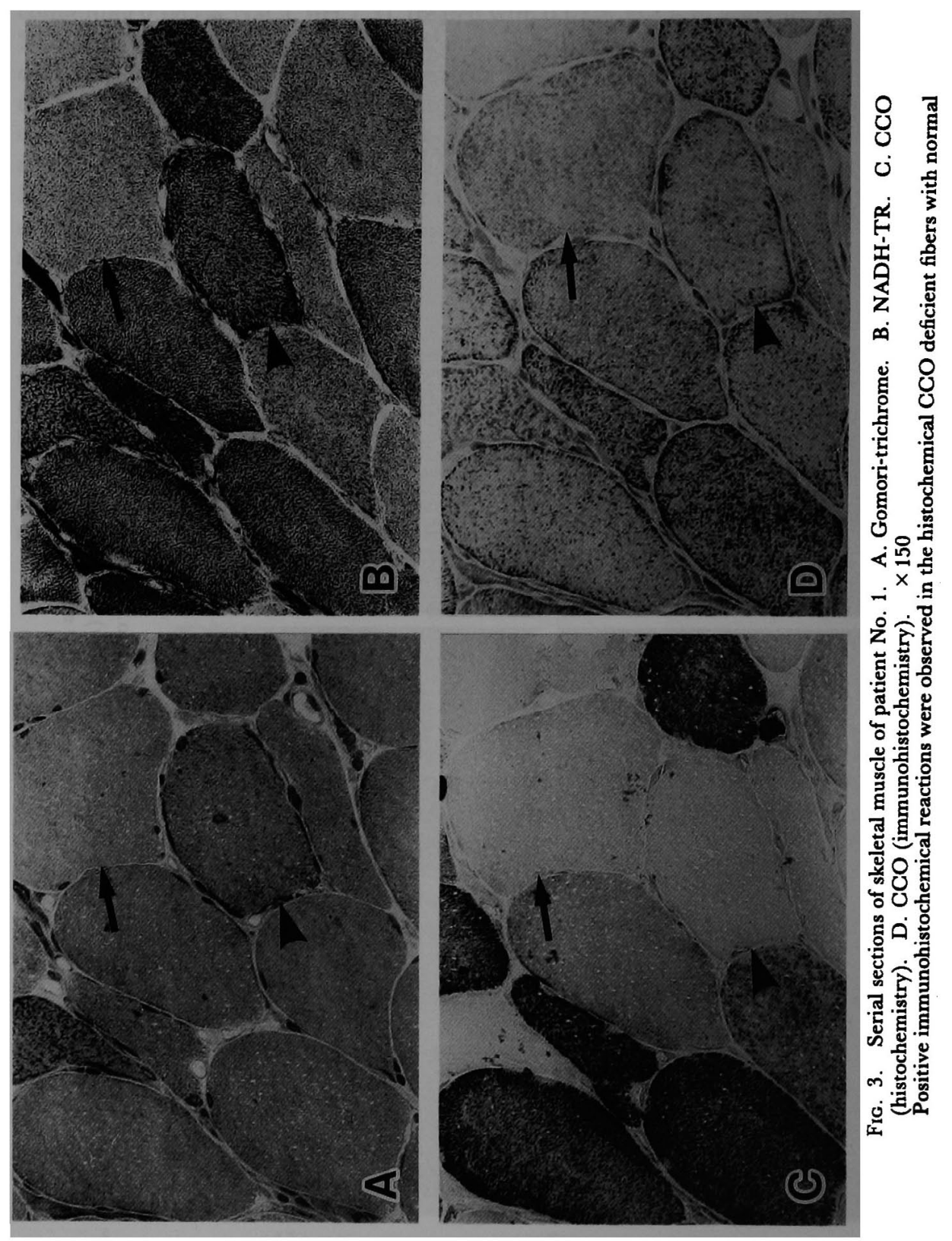



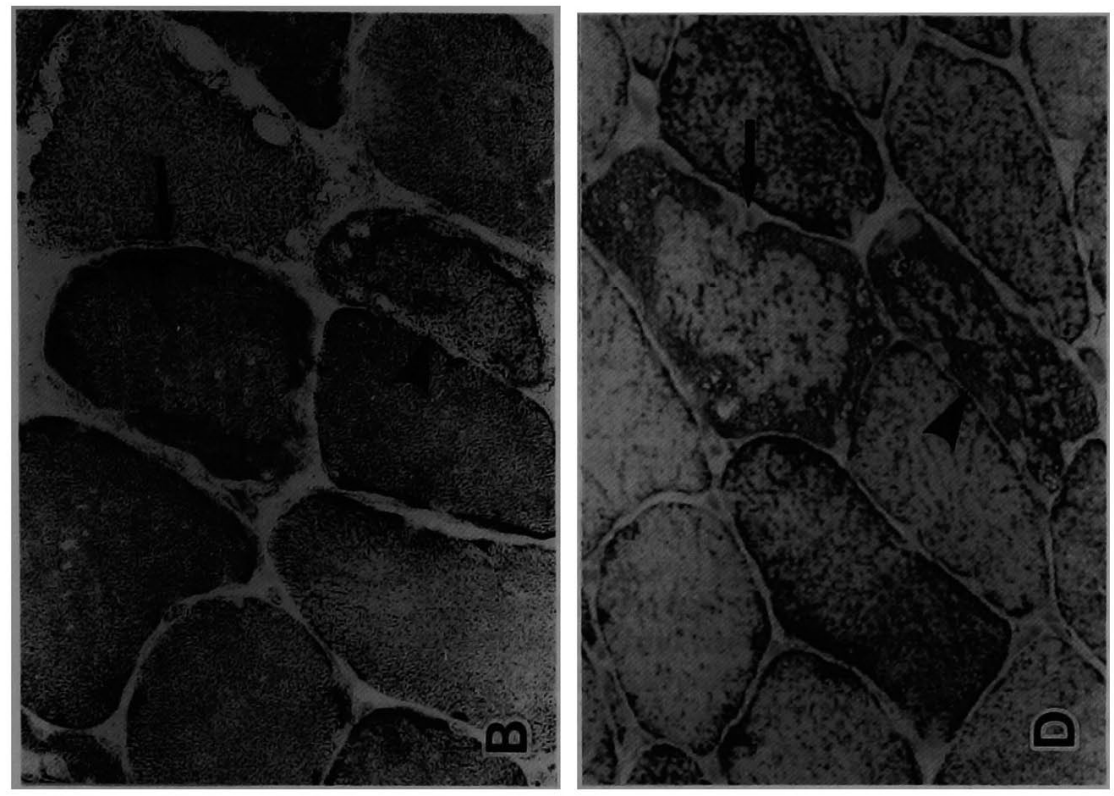

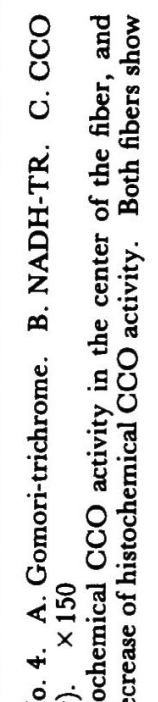
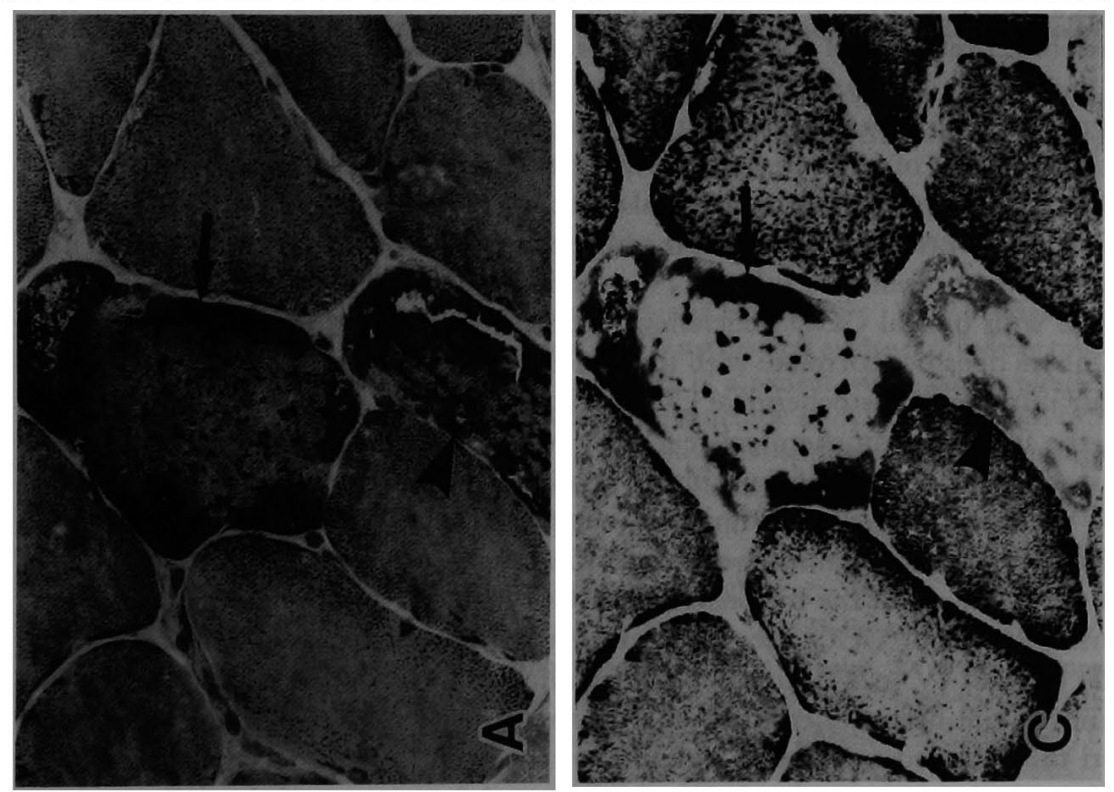

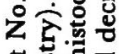

돈

政

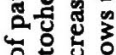

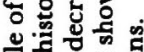

월

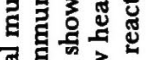

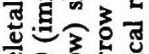

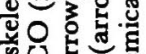

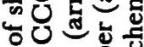

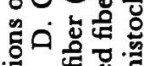

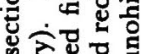
至政

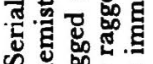

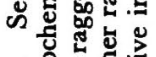
+ 㣢它 它 
activity in ragged red fibers differed from patient to patient, and from fiber to fiber even in the same muscle. Some of the ragged red fibers showed increased CCO activity near the subsarcolemmal area and decreased activity in the center of the fiber (Fig. 4).

Immunohistochemical Staining

We confirmed the specificity of the immunohistochemical staining with monoclonal antibody against subunit IV of CCO since sections incubated with nonimmune mouse serum showed negative reactions (data not shown). In ragged red fibers, the immunohistochemical reaction for the monoclonal antibody was positive even in the histochemical CCO deficient fibers (Fig. 4). On the other hand, the histochemical CCO deficient fibers, other than ragged red fibers, revealed varied immunohistochemical reactions (Figs. 2, 3). The frequency of immunohistochemical CCO deficient fibers varied from 0 to $9 \%$ (Table 2).

\section{DISCUSSION}

Our histochemical studies indicate that there is no correlation between the incidence of ragged red fibers and that of histochemical CCO deficient fibers. The partial deficiency of CCO activity was seen in a fair number of histologically normal fibers. Especially in patient No. 3, almost none of the histochemical CCO deficient fibers were ragged red fibers. These results deviate from a previous study (9) wherein a close correlation between the mitochondrial changes and a negative reaction for CCO activity was noted.

CCO is a complex enzyme composed of at least seven subunits, and it is difficult to evaluate the presence of the subunit protein immunohistochemically using polyclonal antibody. We used the monoclonal antibody against subunit IV of CCO which is synthesized in the cytoplasm under the control of nuclear genes (6). The functional significance of subunit IV of CCO has not been understood yet. In the ragged red fibers, the immunohistochemical reaction for monoclonal antibody against subunit IV of CCO was positive even in the histochemical CCO deficient fibers. This finding suggests the presence of subunit IV protein of CCO in every ragged red fiber in the muscle with partial CCO deficiency. Hence, we think that the formation of ragged red fibers does not correlate with the deficiency of subunit IV protein of CCO.

In a fair number of histochemical CCO deficient fibers found in apparently normal fibers, the immunohistochemical reactivity was deficient. However, in the majority of histochemical CCO deficient fibers, the immunohistochemical reactivity was preserved. While all fibers with immunohistochemical deficient reactivity for subunit IV of CCO had no detectable histochemical CCO activity, subunit IV protein deficiency was not the cause of all the histochemical CCO deficient fibers.

Subunit I to III of CCO are synthesized under the control of mitochondrial DNA, while subunit IV to VII are encoded by nuclear DNA. The exact function of each subunit has not been understood yet. In this study we immunohistochemically investigated the biopsied muscles of patients with partial CCO deficiency using the monoclonal antibody against subunit IV of CCO, and demonstrated the different immuno-reactivity between histochemical CCO deficient fibers in ragged red fibers and those in histologically normal fibers. Various mechanisms may be involved for the occurence of CCO deficiency in muscle fibers. It is necessary to investigate the other 
subunits of CCO to determine the molecular defects of partial or total CCO deficiency.

\section{REFERENCES}

1. Anno, M., Sato, T., Yamauchi, K. and Fukumori, Y.: Diagnosis of mitochondrial myopathy. Biochemical, histochemical and immunohistochemical studies of mitochondrial enzyme in muscle and fibroblast. Neurolog. Med. 24; 143-151, 1986 (in Japanese).

2. Bresolin, N., Zeviani, M., Bonilla, E., Miller, R. H., Leech, R. W., Shanske, S., Nakagawa, M. and DiMauro, S.: Fatal infantile cytochrome $c$ oxidase deficiency. Decrease of immunologically detectable enzyme in muscle. Neurology 35; 802-812, 1985.

3. DiMauro, S., Mendell, J. R., Sahenk, Z., Bachman, D., Scarpa, A., Scofield, R. M. and Reiner, C.: Fatal infantile mitochondrial myopathy and renal dysfunction due to cytochromec-oxidase deficiency. Neurology 30; 795-804, 1980.

4. DiMauro, S., Bonilla, E., Zeviani, M., Nakagawa, M. and Devivo, D. C.: Mitochondrial myopathies. Ann. Neurol. 17; 521-538, 1985.

5. Dubowitz, V. and Brooke, M. H.: Muscle Biopsy-A Modern Approach. Sanders, London, Philadelphia, Toronto 1973.

6. Hare, J. F., Ching, E. and Attardi, G.: Isolation, subunit composition, and site of synthesis of human cytochrome c oxidase. Biochemistry 191; 2023-2030, 1980.

7. Heiman-Patterson, T. D., Bonilla, E., DiMauro, S., Foreman, J. and Schotland, D. L.: Cytochrome-c-oxidase deficiency in a floppy infant. Neurology (NY) 32; 898-900, 1982.

8. Hsu, S.-M., Raine, L. and Fangen, H.: Use of avidin-biotin peroxidase complex (ABC) in immunoperoxidase techniques: a comparison between $\mathrm{ABC}$ and unlabelled antibody (PAP) procedures. J. Histochem. Cytochem. 29; 577-580, 1981.

9. Johnson, M. A., Turnbull, D. M., Dick, D. J. and Sheratt, H. S. A.: A partial deficiency of cytochrome-c-oxidase in chronic progressive external ophthalmoplegia. J. Neurol. Sci. 60; 31$53,1983$.

10. Mitsumoto, H., Aprille, J. R., Wray, S. H., Nemni, R. and Bradley, W. G.: An abnormality of cytochrome-aa ${ }_{3}$ in progressive external ophthalmoplegia (PEO) with morphologic mitochondrial abnormalities. Neurology 31; 58, 1981 (abstract).

11. Müller-Höcker, J., Pongratz, D. and Hübner, G.: Focal deficiency of cytochrome c oxidase in skeletal muscle of patients with progressive external ophthalmoplegia. Virchow's Arch. Path. Anat. 402; 61-71, 1983.

12. Müller-Höcker, J., Stünkel, S., Pongratz, D. and Hübner, G.: Focal deficiency of cytochrome c oxidase and of mitochondrial ATPase with histochemical evidence of loosely coupled oxidative phosphorylation in a mitochondrial myopathy of a patient with bilateral ptosis. An enzyme histochemical, immunohistochemical and fine structural study. J. Neurol. Sci. 69; 27-36, 1985.

13. Nakagawa, M. and Miranda, A.F.: A monoclonal antibody against cytochrome $c$ oxidase distinguishes cardiac and skeletal muscle mitochondria. Exp. Cell. Res. 168; 44-52, 1987.

14. Seligman, A. M., Karnovsky, M. J., Wasserkrug, H. L. and Hanker, J. S.: Non-droplet ultrastructural demonstration of cytochrome oxidase activity with a polymerizing osmiophilic reagent, diaminobenzidine (DAB). J. Cell. Biol. 38; 1-14, 1968.

15. Van Biervliet, J. P. A. M., Bruinsvis, L., Ketting, D., DeBree, P. K., van der Heiden, C., Wadman, S., Willems, J. L., Bookelman, H., van Haelst, U. and Monnens, L. A. H.: Hereditary mitochondrial myopathy with lactic acidemia, a De Toni-Fanconi-Debre syndrome, and a defective respiratory chain in voluntary striated muscle. Pediatr. Res. 11; 1088-1093, 1977.

16. Zeviani, M., Nonaka, I., Bonilla, E., Okino, E., Moggino, M., Jones, S. and DiMauro, S.: Fatal infantile mitochondrial myopathy and renal dysfunction caused by cytochrome $\mathrm{c}$ oxidase deficiency. Immunological studies in a new patient. Ann. Neurol. 17; 414-417, 1985. 